Published in final edited form as:

Nature. ; 483(7388): 164-165. doi:10.1038/483164a.

\title{
Gorilla gorilla gorilla
}

\author{
Richard A. Gibbs and Jeffrey Rogers \\ Human Genome Sequencing Center, Department of Molecular and Human Genetics, Baylor \\ College of Medicine, Houston, Texas 77030, USA
}

Humans and the apes are the living representatives of the superfamily Hominoidea, which also contains many extinct species. Deciphering the evolutionary relationships between these species is an essential step in our understanding of the biological richness of the planet, and of our own evolutionary history. The draft assembly of the whole-genome sequence of a female western lowland gorilla (Gorilla gorilla) named Kamilah (Fig. 1), presented by Scally et al. ${ }^{1}$ on page 169 of this issue, provides insight into how a single hominid lineage separated into the extant human, chimpanzee and gorilla branches.

The authors' genome assembly, made possible by the advent of cheaper next-generation sequencing methods, is a much-anticipated addition to more than five-year-old Sanger sequence data on the gorilla genome. The latest assembly, like other contemporary mammalian genome data sets (except for human and mouse), has some gaps and shortcomings. Yet it is revelatory. The standard view of the primate evolutionary tree is that chimpanzees and humans share a more recent common ancestor with each other than either shares with gorillas. Accordingly, the most closely related sequence for any human gene should be found in the chimpanzee. However, Scally and colleagues' demonstrate that, although this is true for most genes, large fractions of the ape genomes contradict this simple pattern.

Molecular phylogenetics uses comparative analyses of DNA sequences to determine relatedness among species. Usually, a single individual of each species is sequenced, so genetic diversity within a species is overlooked. When the time between successive evolutionary branch points, or speciation events, is relatively long, the between-species genetic differences that accumulate after a single lineage divides into two descendent branches will stand out against the background of within-species variation. But if two or more evolutionary divergence events occur close together in time, the genetic variation present in the last common ancestor may be sorted randomly into the descendent lineages. In this model, different segments of a genome may have different phylogenetic relationships. This process, which leads to conflicting evolutionary trees for different genes, is called incomplete lineage sorting (ILS; Box 1).

Previous molecular-genetic studies ${ }^{2,3}$ of humans, chimpanzees and gorillas show that the three lineages separated over a relatively short period of time, creating the opportunity for ILS. Scally and colleagues' whole-genome study verifies and substantially extends this analysis. They found that for $70 \%$ of the genomes of the three species, the chimpanzee sequences are more similar to the corresponding sequences in humans than to those in the gorilla, as expected. But for the remaining $30 \%$ of the genome, gorilla sequences share 
closer similarity with either human or chimpanzee sequences than these two share with each other. The authors also observed these ILS patterns reflected in relative levels of gene expression in the three species.

Other factors, such as gene flow between species after their initial divergence (Box 1), may also have contributed to these surprising relationships. There is evidence ${ }^{4}$ for such gene flow between Neanderthals and the lineage that ultimately produced modern humans, and between morphologically differentiated non-human primate groups that are often considered separate species, such as macaques ${ }^{5,6}$ and baboons ${ }^{7,8}$. Thus, the data presented by Scally and colleagues ${ }^{1}$ support a growing challenge to the idea that primate species diversity always increases through a process in which one species divides rapidly and irreversibly into two genetically isolated daughter species.

New calculations to establish when the gorilla, chimpanzee and human lineages diverged are now possible. By combining data on DNA-sequence differences between species with estimates of DNA-mutation rates operative millions of years ago, Scally and colleagues calculate the human-chimpanzee and (human+chimpanzee)-gorilla branch points to be 3.7 million and 5.95 million years ago, respectively. These dates conflict with some fossil evidence, but more complex calculations that allow for mutation rates that change with time - a reasonable possibility owing to changes in the average length of generations and other parameters - may resolve this problem.

An overarching question is how these three divergent lineages each acquired their unique morphological and behavioural characteristics — including how one lineage became human. The new gorilla data suggest that a large proportion of the human genome was under positive selection pressure during the period of initial isolation from our close relatives, indicating that natural selection drove evolutionary innovation and change. Scally and colleagues' study adds to our ability to reconstruct those selective forces that acted on each lineage.

The report ${ }^{1}$ also provides information on genetic diversity within the Gorilla genus. The authors compared Kamilah's genome with sequence data from two other western lowland gorillas and an eastern lowland gorilla (Gorilla beringei graueri), and found substantial genetic differentiation within the genus, consistent with previous data ${ }^{9}$. The genus is currently classified into four subspecies, two for each of the two species, but the variation identified by Scally et al. suggests further analysis of genetic diversity in gorillas is warranted.

Gorillas have inspired awe and scientific interest throughout history. The sequencing of the gorilla genome adds valuable information to our understanding of these remarkable animals and our evolutionary relationship to them - revealing a closer connection between our genome and that of the gorilla than was previously appreciated. Sadly, the future of gorillas is uncertain, with all populations under severe human-induced pressure, and the mountain gorillas especially close to extinction. Their fate is inextricably linked to our choices, and these extraordinary primates deserve our utmost efforts at conservation. To quote from 
American author Daniel Quinn's novel Ishmael, "with man gone, will there be hope for gorilla?" or, even more soberingly, "with gorilla gone, will there be hope for man?"

\section{References}

1. Scally A, et al. Nature. 2012; 483:169-175. [PubMed: 22398555]

2. Rogers J. Am. J. Phys. Anthropol. 1994; 94:81-88. [PubMed: 8042707]

3. Ruvolo M. Mol. Biol. Evol. 1997; 14:248-265. [PubMed: 9066793]

4. Green RE, et al. Science. 2010; 328:710-722. [PubMed: 20448178]

5. Tosi AJ, Morales JC, Melnick DJ. Int. J. Primatol. 2002; 23:161-178.

6. Osada N, et al. Mol. Ecol. 2010; 19:2884-2895. [PubMed: 20579289]

7. Alberts SC, Altmann J. Am. J. Primatol. 2001; 53:139-154. [PubMed: 11283975]

8. Jolly CJ, Burrell AS, Phillips-Conroy JE, Bergey C, Rogers J. Am. J. Primatol. 2011; 73:291-303. [PubMed: 21274900]

9. Thalmann O, Fischer A, Lankester F, Pääbo S, Vigilant L. Mol. Biol. Evol. 2007; 24:146-158. [PubMed: 17065595] 


\section{BOX 1 How incongruities in phylogenetic trees can arise}

Scally and colleagues ${ }^{1}$ found that in $30 \%$ of the western-lowland-gorilla genome, the DNA sequences are more similar to the corresponding sequences from the human or chimpanzee genomes than the sequences of these two species are to each other although humans and chimpanzees are expected to have shared a more recent common ancestor with each other than either does with gorillas. Such inconsistencies between evolutionary relationships can result from various processes. a, One possible mechanism is incomplete lineage sorting. In this process, an ancestral species (black) divides into two descendent genetic lineages (green and red) and, soon after, one of those descendent lineages divides again (red and orange). The red and orange lineages are expected to be more genetically similar to one another than either is to the green lineage. However, if the ancestral species contained a gene with two alternative sequence variants (AB), either or both variants may be transmitted into the descendants. Over time, the descendent species will lose one of the two gene variants. Here, incomplete lineage sorting has resulted in the red species being more genetically similar to the green species (both AA) than to the orange species (BB) at this particular gene. $\mathbf{b}$, Gene flow is another mechanism by which relationships between specific DNA sequences can fail to match the larger relationships between species. In this case, a newly evolved gene variant (C) is transferred from one genetic lineage to another by interbreeding that occurs after evolutionary separation has begun, but before complete genetic isolation is achieved.

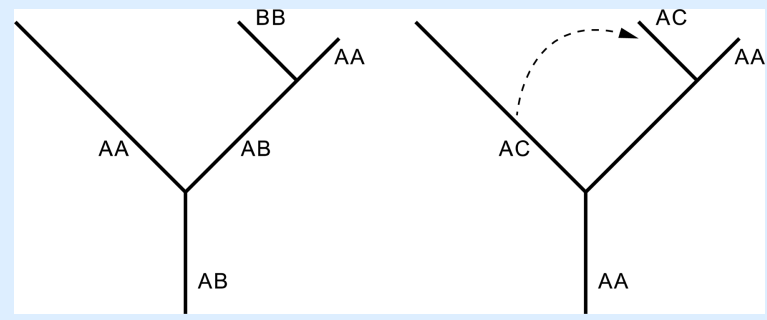




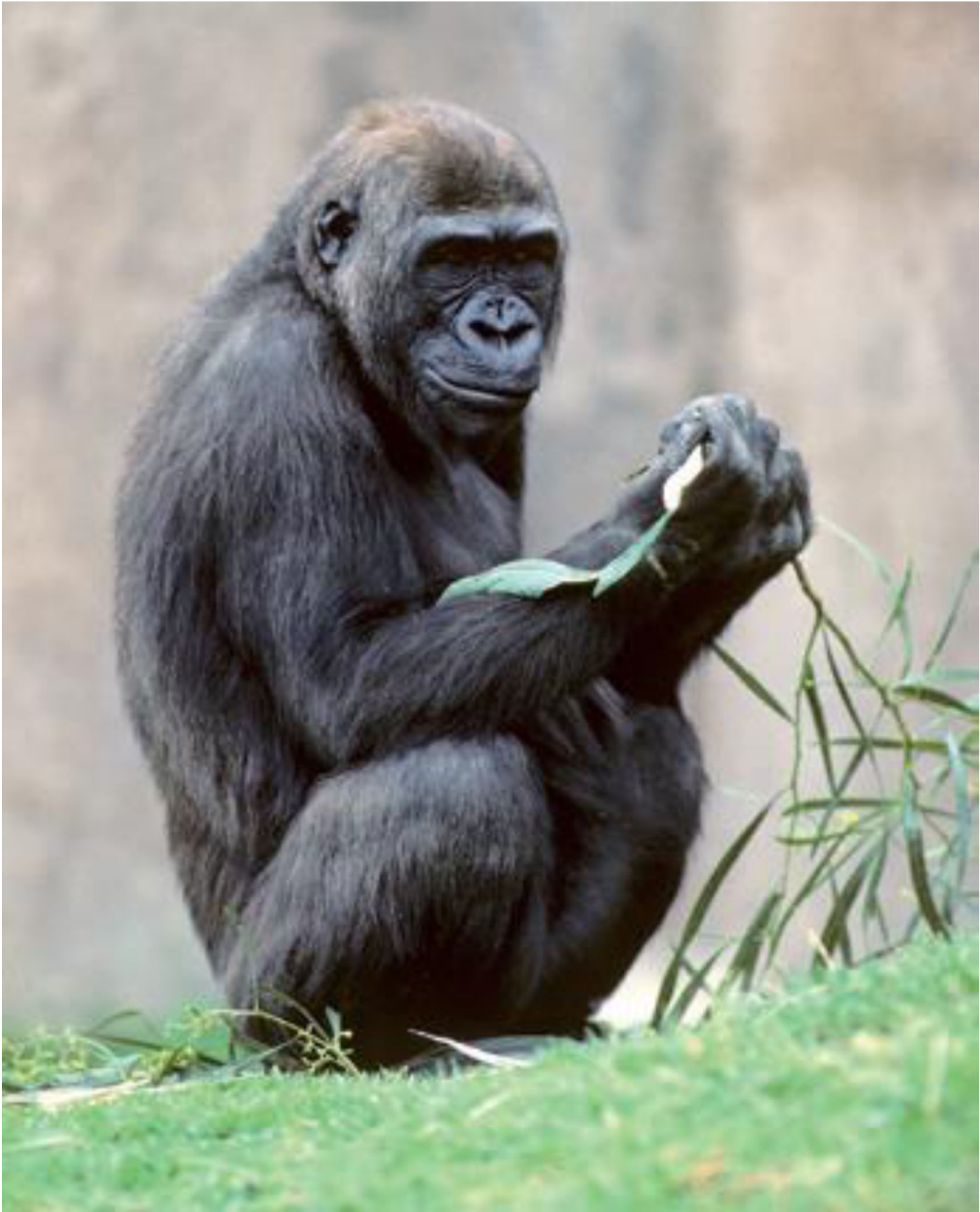

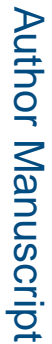

Figure 1. Kamilah the gorilla

Scally et al. ${ }^{1}$ report the whole-genome sequence of this western lowland gorilla 